Mini Review

\title{
Cancer Immunotherapy: Managing Amino Acids during Forced Aetopy
}

\author{
Michael J Dochniak* \\ Alleam, LLC, Minnesota USA
}

\begin{abstract}
Cancer patients of all ages experience great satisfaction with initial positive results from first-line therapy. Adjuvant therapy helps decrease the risk of cancer recurring or metastasizing. This review discusses forced atopy as cancer immunotherapy and explores managing select amino acids to starve metastatic cells during forced atopy (many allergies).
\end{abstract}

\section{Introduction}

A common goal in oncology is a treatment protocol that minimizes risk and maximizes success. The overall survival rate of children with solid tumor metastasis (Stage IV) has shown little improvement in that the extent of the relocation of metastatic cells, and their progression, is not well understood. To better understand the limits of metastasis, an alternative treatment strategy is proposed; based on maladaptive immunity. A skin cream having natural and recombinant allergens, and associated with immunologic adjuvants, is vectored into the cancer patient by topical dermal absorption. After that, humoral immunity increases the expression of cross-reactive immunoglobulin-E (IgE) primed effector cells, designed to decrease the incidence and prevalence of endogenous proteins that support the metastatic environment. ${ }^{1}$

Cancer recurrence or metastasis after first-line therapies is life-threatening. In metastasis, metastatic cells from a primary tumor spread throughout the body, forming secondary tumors that aggressively grow and often cause death. Approximately $90 \%$ of cancer deaths are due to metastasis. ${ }^{2}$

The management of dietary amino acids during forced atopy may be a vital treatment protocol. Some micronutrients and foodstuff components have specific roles in the development and main tenance of an effective immune systemthroughout the life course or in reducing chronic inflammation. For example, the amino acid arginine is essential for the generation of nitric oxide by macrophages, and the micronutrients vitamin $\mathrm{A}$ and zinc regulate cell division are vital for a successful proliferative response within the immune system. ${ }^{3}$ Atopy increases the use of amino acids to provide building blocks for the biosynthesis of effector cells and immunoglobulins. ${ }^{4}$ Amino acid competition between immune cells and cancer cells can influence their growth, survival, and function. A fierce competition likely exists between cells in the tumor microenvironment, as demand for resources in this niche is high. There is evidence for the metabolic interplay between cancer cells and immune cells. ${ }^{5}$

During forced atopy, an understanding of dietary amino acid competition between cancer cells and immune cells may lead to improvements in starving cancer. The amino acid arginine can enhance the immune response against cancer, allowing an expanded population of immune cells to consume glutamine. ${ }^{6}$ In continuation, increased glutamine utilization by immune cells may affect glutamine availability for metastatic cells, inhibiting a metastatic cell's ability to convert glutamine into biomass. ${ }^{7}$ This review explores the management of select amino acids to starve metastatic cells during forced atopy.

\begin{tabular}{|c|l|}
\hline Quick Response Code: & *Corresponding author: Michael J Dochniak, Alleam, LLC, Minnesota USA \\
Received: 18 November, 2020 & Published: 11 December, 2020 \\
& Citation: Michael J Dochniak. Cancer Immunotherapy: Managing Amino Acids during Forced \\
\hline
\end{tabular}




\section{Discussion}

Nutrition therapy helps cancer patients keep healthy body weight, maintain strength, keep body tissue healthy, and decrease side effects both during and after treatment. Nutrition is a process in which food is taken in and used by the body for growth, to keep the body healthy, and to replace tissue. Good nutrition is vital for good health. Eating the right kinds of foods before, during, and after cancer treatment can help the patient feel better and stay stronger. A healthy diet includes eating and drinking enough of the foods and liquids that have vital nutrients (vitamins, minerals, protein, carbohydrates, fat, and water) the body needs. ${ }^{8}$

Nutrition as adjuvant therapy supports the immune system and lowers the risk of cancer reoccurrence or metastasis. Can forced atopy, while managing select dietary amino acids, inhibit tumor growth, and prolong survival? Hyper-allergenic skin creams interfere with processes that disrupt metastatic cells through immune-metabolic interference. Forced atopy as cancer immunotherapy increases chronic inflammation. Cancer suppression during forced atopy is suspected with IgEantibodies in that they are biologically active despite low concentration in the bloodstream, approximately one-thousandth of a percent. IgE bind to high-affinity receptors on the surface of effector cells (e.g., mast cells, basophils, and eosinophils) to provide IgE-primed effector cells, extremely sensitive to allergens, producing chronic inflammatory reactions. ${ }^{9}$ Starving cancer is a formidable task. The complex interplay of amino acids between cancer cells and immune cells is often not clearly understood. Research indicates that amino acids in diet could starve cancer. ${ }^{10}$

\section{Glutamine}

The cancer effect of managing dietary glutamine during forced atopy is unclear. In cancer research, there is evidence of an essential role for glutamine in tumors and that a variety of factors, including tissue type, the underlying cancer genetics, the tumor microenvironment, and other variables such as diet and host physiology collectively influence the role of glutamine in cancer. ${ }^{11}$ Cancer cells can engage in glutamine metabolism that exceeds the requirement for protein and nucleotide synthesis. ${ }^{12}$ Immune system research shows that glutamine is essential for the activation of cells involved in both innate and adaptive immunity. Prolonged or chronic states of severe inflammation require large quantities of dietary glutamine. ${ }^{13}$ Glutamine is by far the most abundant free amino acid in plasma and tissues in humans. Plasma concentrations vary between 400 and $600 \mu \mathrm{mol} / \mathrm{l}$ and tissue concentrations between 2 and $20 \mathrm{mmol} / \mathrm{l}$ intracellular water ${ }^{14}$ Forced atopy is a cancer immunotherapy that may produce prolonged or chronic states of severe inflammation. Dietary sources of glutamine include protein-rich foods like beef, chicken, fish, seafood, dairy products, eggs, vegetables like beans, beets, cabbage, spinach, carrots, parsley, vegetable juices, wheat, papaya, Brussel sprouts, celery, kale, and fermented foods like miso.

\section{Arginine}

The cancer effect of increasing dietary arginine during forced atopy is unclear. Cancer research shows that arginine de novo synthesis is not enough to compensate for high nutritional needs, forc- ing them to rely on an extracellular supply of arginine..$^{15}$ Immune system research shows that L-arginine boosts adaptive immunity by influencing t-cell proliferation, differentiation, and survival. Helper t-cells are considered vital for almost all adaptive immune responses. ${ }^{16}$ Helper t-cells assist in the activation of b-cells that can differentiate into plasma cells that produce immunoglobulin-E antibodies. ${ }^{17}$ The usual range of L-arginine plasma levels is $81.6 \pm 7.3 \mathrm{mmol} / \mathrm{L}$ in young men and $113.7 \pm 19.8 \mu \mathrm{mol} / \mathrm{L}$ in men, as compared with $72.4 \pm 6.7 \mu \mathrm{mol} / \mathrm{L}$ in young women and $88.0 \pm 7.8$ $\mu \mathrm{mol} / \mathrm{L}$ in women. ${ }^{18}$ Many foods are natural sources of arginine like nuts (like walnuts, hazelnuts, pecans, peanuts, almonds, cashews, pine nuts, and Brazil nuts), seeds (like watermelon, pumpkin, sesame, sunflower), oats, corn, cereals, buckwheat, brown rice, and chocolate. Animal sources of arginine include meat, dairy products, and eggs. ${ }^{19}$

\section{Serine}

The cancer effect of increasing dietary serine during forced atopy is unclear. Cancer research shows that dietary restriction of serine may have antitumor effects. ${ }^{20}$ Immune system research shows that serine metabolism is vital for optimal T-cell proliferation by fueling one-carbon metabolism and nucleotide biosynthesis. ${ }^{21} \mathrm{Di}-$ etary sources of L-serine include soy products, sweet potatoes, eggs, meat, and some edible seaweed..$^{22}$

\section{Methionine}

The cancer effect of managing dietary methionine during forced atopy is unclear. Cancer research shows that methionine restriction inhibits cancer cell growth. Methionine is vital for cancer cell growth and metabolism. ${ }^{23}$ Methionine deprivation suppresses triple-negative breast cancer metastasis in vitro and in vivo. ${ }^{24}$ Immune system research shows that T-helper cells import the amino acid methionine to synthesize new proteins and to provide the methyl groups needed for the methylation of RNA and DNA that drives T-cell proliferation and differentiation. ${ }^{25}$ The recommended daily intake for methionine is $10.4 \mathrm{mg}$ per kilogram of body weight or $4.5 \mathrm{mg}$ per pound. Foods having high levels of methionine include ground turkey, beef (skirt steak), tuna, lean pork chops, firm tofu, milk, low-fat ricotta cheese, brazil nuts, white beans, and whole grains like quinoa ${ }^{26}$

\section{Alanine and carnosine}

The cancer effect of managing dietary alanine during forced atopy is unclear. Alanine is a substrate for the hepatic synthesis of glucose, a significant energy substrate for leucocytes. ${ }^{27}$ Cancer research shows that pancreatic stellate cells, which form the stromal compartment of pancreatic cancers, secrete large amounts of alanine to support the metabolic activity of cancer cells. ${ }^{28}$ Immune system research shows beta-alanine exerts immunoregulatory effects by activating both T and B-cells. ${ }^{29}$ Foods that have high levels of alanine include meat, fish, and poultry. ${ }^{30}$ The cancer effect of increasing dietary carnosine during forced atopy may be beneficial. Carnosine (beta-alanyl-L-histidine) is a dipeptide molecule made up of the amino acids beta-alanine and histidine; muscle and brain tissue have high concentrations. Cancer research shows that L-carnosine can restrict the behavior of ovarian cancer cells, related to 
the cancerous phenotype of senescent human peritoneal mesothelial cells. ${ }^{31}$ Immune system research shows that carnosine exerts immunoregulatory effects by activating both T and B cells. ${ }^{32}$ Foods having high levels of carnosine include meat, fish, and poultry. A vegetarian or vegan diet provides little or no carnosine in comparison to the amounts found in a meat diet. ${ }^{33}$

\section{Tryptophan}

The cancer effect of limiting dietary tryptophan during forced atopy may be beneficial. Cancer research shows that tumors increase their consumption of tryptophan to evade immune control. ${ }^{34}$ Immune system research shows that tryptophan metabolites along the kynurenine pathway induce immunosuppression involving apoptosis of effector immune cells, which tumors use to escape an immune response. ${ }^{35}$ The intake of tryptophan for many individuals is approximately 900 to $1000 \mathrm{mg}$ daily. The recommended daily allowance for adults is estimated to be between $250 \mathrm{mg} /$ day to 425 $\mathrm{mg} /$ day, which translates to a dietary intake of 3.5 to $6.0 \mathrm{mg} / \mathrm{kg}$ of body weight per day. ${ }^{36}$ Tryptophan is present in most protein-based foods or dietary proteins. It is particularly plentiful in chocolate, oats, dried dates, milk, yogurt, cottage cheese, red meat, eggs, fish, poultry, sesame, chickpeas, almonds, sunflower seeds, pumpkin seeds, buckwheat, spirulina, and peanuts. ${ }^{37}$

\section{Glutamic acid}

The cancer effect of limiting dietary glutamic acid during forced atopy may be beneficial. Cancer research shows glutamic acid and the sodium salt of glutamic acid (i.e., glutamate) modulate cancer cell development, proliferation, and metastasis through regulating cell signaling pathways. Glutamate activates glutamate receptors on cancer cells and results in malignant growth. ${ }^{38}$ Immune system research shows that glutamic acid may inhibit cross-reactivity of IgEprimed effector cells reducing the efficacy of forced atopy as cancer immunotherapy. Scientists showed that pre-absorption of serum with glutamic acid resulted in inhibition of IgE reactivity to both inhalable and food allergens, indicating that the carboxyl group of these amino acids is vital in IgE-epitope interactions..$^{39}$ Foods high in glutamic acid include meats, poultry, fish, eggs, wheat, beans, seeds, tomatoes, cheese, mushrooms, wheat gluten, and dairy products. ${ }^{40}$

\section{Monosodium glutamate (MSG)}

The cancer effect of limiting dietary monosodium glutamate during forced atopy may be beneficial. Monosodium glutamate (MSG) is the sodium salt of glutamic acid. Cancer research indicates there is no conclusive evidence linking the consumption of MSG to a cause of cancer or an increased risk of cancer. Immune system research shows that exposure to increasing MSG concentrations (1-100 mM) showed a dose-dependent effect on B-cell viability. Glutamate induced apoptosis in both memory and naive B-cell populations is affected by metabotropic glutamate receptor (mGluR) 7 receptors. ${ }^{41}$ MSG is a flavor enhancer, used for over 100 years, and is found naturally in tomatoes and cheese.

\section{Asparagine}

The cancer effect of managing dietary asparagine during forced atopy is unclear. Cancer research shows that asparagine availabil- ity promotes the formation of lung metastasis in breast cancer by promoting epithelial-to-mesenchymal transition. ${ }^{42}$ Asparagine can promote the survival of cancer cells in response to glutamine withdrawal. ${ }^{43}$ Immune system research shows that exogenous asparagine, a nonessential amino acid, is vital for naive T-cells to synthesize new proteins following TCR engagement, which is consistent with the notion that nutrient availability is essential for proper T-cell activation. ${ }^{44}$ Foods rich in asparagine include dairy, whey, beef, poultry, eggs, fish, seafood, asparagus, potatoes, legumes, nuts, seeds, soy, and whole grains. Foods low in asparagine include most fruits and vegetables.

\section{Conclusion}

Starving cancer is a formidable task. The adjuvant therapy described herein discusses managing select amino acids to starve metastatic cells during forced atopy. Research efforts should continue to explore the complex interplay of dietary amino acids, cancer cells, and immune cells to improve cancer treatment outcomes for children and adults.

\section{Author disclosure}

Michael J. Dochniak is cofounder of Alleam, LLC, Minnesota, USA. This commentary contains a discussion of an unapproved/ investigative hyper-allergenic skin cream to inhibit cancer reoccurrence or metastatic cancer.

\section{Acknowledgments}

None.

\section{Funding}

None.

\section{Conflicts of interest}

Michael J. Dochniak is co-founder and CTO of Alleam, LLC. Minnesota, USA.

\section{References}

1. Dochniak MJ. Pediatric Metastasis and cIgE Antibodies. Open J Pediatr Neonatal Care. 2020;5(1):001-003.

2. Seyfried TN, Huysentruyt LC. On the origin of cancer metastasis. Critical reviews in oncogenesis. 2013;18(1-2):43-73.

3. Childs CE, Calder PC, Miles EA. Diet and Immune Function. Nutrients. 2019;11(8):1933.

4. Janeway CA, Travers $\mathrm{P}$, Walport $\mathrm{M}$, et al. Immunobiology: The Immune System in Health and Disease. $5^{\text {th }}$ edn. New York: Garland Science; 2001.

5. Chang CH, Qiu J, O'Sullivan D, et al. Metabolic Competition in the Tumor Microenvironment Is a Driver of Cancer Progression. Cell. 2015;162(6):1229-1241.

6. Geiger R, Rieckmann JC, Wolf $\mathrm{T}$, et al. L-Arginine Modulates $\mathrm{T}$ Cell Metabolism and Enhances Survival and Anti-tumor Activity. Cell. 2016;167(3):829-842.e13.

7. Tian Y, Du W, Cao S, et al. Systematic analyses of glutamine and glutamate metabolisms across different cancer types. Chin J Cancer. 2017;36:88.

8. Soldati L, Di Renzo L, Jirillo E, et al. The influence of diet on anti-cancer immune responsiveness. J Transl Med. 2018;16(1):75.

9. Dochniak MJ. Maladaptive immunity and metastasizing cancer. Cancer Med J. 2020;3:31-34. 
10. Cancer Research UK. Amino acids in diet could be key to starving cancer. Science Daily. 2017.

11. Cluntun AA, Lukey MJ, Cerione RA, et al. Glutamine Metabolism in Cancer: Understanding the Heterogeneity. Trends Cancer. 2017;3(3):169-180.

12. De Berardinis RJ, Mancuso A, Daikhin E, et al. Beyond aerobic glycolysis: transformed cells can engage in glutamine metabolism that exceeds the requirement for protein and nucleotide synthesis. Proc Natl Acad Sci USA. 2007;104(49):19345-19350.

13. Soeters PB, Grecu I. Have we enough glutamine and how does it work? A clinician's view. Ann Nutr Metab. 2012;60(1):17-26.

14. Cruzat V, Macedo Rogero M, Noel Keane K, et al. (Glutamine: Metabolism and Immune Function, Supplementation and Clinical Translation. Nutrients. 2018;10(11):1564.

15. Al-Koussa H, El Mais N, Maalouf $\mathrm{H}$, et al. Arginine deprivation: a potential therapeutic for cancer cell metastasis? A review. Cancer Cell Int. 2020;20:150

16. Geiger R, Rieckmann JC, Wolf T, et al. L-Arginine Modulates T Cell Metabolism and Enhances Survival and Anti-tumor Activity. Cell. 2016;167(3):829-842.e13.

17. Alberts B, Johnson A, Lewis J, et al. Molecular Biology of the Cell. $4^{\text {th }}$ edn. New York: Garland Science; 2002.

18. Böger RH. The pharmacodynamics of L-arginine. Alternative therapies in health and medicine. 2014;20(3):48-54.

19. Medical News Today. Jenna Fletcher. Which foods are high in arginine? 2018.

20. Maddocks ODK, Athineos D, Cheung EC, et al. Modulating the therapeutic response of tumours to dietary serine and glycine starvation. Nature. 2017;544(7650):372-376.

21. Ma EH, Bantug G, Griss T, et al. Serine Is an Essential Metabolite for Effector T Cell Expansion. Cell Metab. 2017;25(2):345-357.

22. Nutrition Data, Foods highest in serine.

23. Wanders D, Hobson K, Ji X. Methionine Restriction and Cancer Biology. Nutrients. 2020;12(3):684.

24. Jeon H, Kim JH, Lee E, et al. Methionine deprivation suppresses triplenegative breast cancer metastasis in vitro and in vivo. Oncotarget. 2016;7(41):67223-67234

25. Klein Geltink RI, Pearce EL. The importance of methionine metabolism. Elife. 2019;8:e47221.

26. Whitbread. High Methionine Foods. 2020.

27. Newsholme P, Newsholme EA. Rates of utilization of glucose, glutamine and oleate and formation of end-products by mouse peritoneal macrophages in culture. Biochem J. 1989;261(1):211-218.
28. Sousa CM, Biancur DE, Wang X, et al. Pancreatic stellate cells support tumour metabolism through autophagic alanine secretion [published correction appears in Nature. Nature. 2016;536(7617):479-483.

29. Nagai K, Suda T. Immunoregulative effects of carnosine and beta-alanine. Nihon Seirigaku Zasshi. 1986;48(6):564-71.

30. Medical News Today. What to know about essential amino acids.

31. Mikuła PietrasikJ, Książek K. L-Carnosine Prevents the Pro-cancerogenic Activity of Senescent Peritoneal Mesothelium Towards Ovarian Cancer Cells. Anticancer Res. 2016;36(2):665-671.

32. Sousa CM, Biancur DE, Wang X, et al. Pancreatic stellate cells support tumour metabolism through autophagic alanine secretion [published correction appears in Nature. Nature. 2016;536(7617):479-483.

33. Hipkiss AR. Carnosine and its possible roles in nutrition and health. $A d v$ Food Nutr Res. 2009;57:87-154.

34. Prendergast GC. Cancer: Why tumours eat tryptophan. Nature. 2011;478(7368):192-194.

35. Badawy AA. Tryptophan Metabolism: A Versatile Area Providing Multiple Targets for Pharmacological Intervention. Egypt J Basic Clin Pharmacol. 2019;9:10.32527/2019/101415.

36. Richard DM, Dawes MA, Mathias CW, et al. L-Tryptophan: Basic Metabolic Functions, Behavioral Research and Therapeutic Indications. Int J Tryptophan Res. 2009;2:45-60.

37. Friedman M. Analysis, Nutrition, and Health Benefits of Tryptophan. Int J Tryptophan Res. 2018;11:1178646918802282.

38. Yi H, Talmon G, Wang J. Glutamate in cancers: from metabolism to signaling. J Biomed Res. 2019;34(4):260-270.

39. Deus-de-Oliveira N, Felix SP, Carrielo-Gama C, et al. Identification of critical amino acids in the IgE epitopes of Ric c 1 and Ric c 3 and the application of glutamic acid as an IgE blocker. PLoS One. 2011;6(6):e21455.

40. Nutrition Data, Foods highest in glutamin acid.

41. Jovic Z, Veselinovic M, Vasic K, et al. Monosodium glutamate induces apoptosis in naive and memory human B cells. Bratisl Lek Listy. 2009;110(10):636-640.

42. Knott SRV, Wagenblast E, Khan S, et al. Asparagine bioavailability governs metastasis in a model of breast cancer. Nature. 2018;554(7692):378381.

43. Zhang J, Fan J, Venneti S, et al. Asparagine plays a critical role in regulating cellular adaptation to glutamine depletion. Mol Cell. 2014;56(2):205218.

44. Torres A, Luke JD, Kullas AL, et al. Asparagine deprivation mediated by Salmonella asparaginase causes suppression of activation-induced $\mathrm{T}$ cell metabolic reprogramming. J Leukoc Biol. 2016;99(2):387-398. 